\title{
РОЛЬ ШКАЛ РАSS И GАРP В ОПРЕДЕЛЕНИИ ЗЛОКАЧЕСТВЕННОГО ПОТЕНЦИАЛА ФЕОХРОМОЦИТОМЫ. КЛИНИЧЕСКИЙ СЛУЧАЙ
}

\author{
1 Мотренко А.Ю., ' Воронкова И.А., ${ }^{1}$ Бритвин Т.А., ${ }^{1}$ Иловайская И.А., ${ }^{1}$ Гуревич Л.Е.
}

'ГБУЗ МО «Московский областной научно-исследовательский клинический институт им. М. Ф. Владимирского»

Согласно 4 изданию классификации ВОЗ опухолей эндокринных органов (2017 г.), все феохромоцитомы (ФХЦ) относят к злокачественным опухолям (ICD-O code 8700/3) на основании того, что они обладают метастатическим потенциалом. В настоящее время для оценки риска прогрессирования ФХЦ предложены две шкалы: PASS (Pheochromocytoma of the Adrenal gland Scaled Score 2002 г) и GAPP (Grading system for adrenal pheochorocytoma and paraganglioma 2014). Однако, однозначного мнения в отношении их прогностической ценности нет, в результате чего они не нашли широкого применения в клинической практике. Представляем клинический случай, который демонстрирует возможность оценки прогноза ФХЦ с использованием шкал PASS и GAPP.

ЦЕЛЬ: оценить возможности шкал PASS и GAPP в определении злокачественного потенциала феохромоцитомЫ.

МАТЕРИАЛЫ И МЕТОДЫ: ПацИеНт Б. 47 лет НаблюДался в ГБУЗ МО МОНИКИ в СвязИ С ПостоянНЫМ повышением артериального давления (АД) мах до 220/110 мм рт.ст., сопровождающееся головной болью, покраснением лица и шеи, не купируемое приемом антигипертензивных препаратов. По данным мультиспиральной компьютерной томографии (МСКТ) с контрастным усилением (КУ), в правом надпочечнике определяется образование размерами 62×52×67 мм, с четкими ровными контурами, неоднородной структуры и плотностью 12-50 ед.Н в нативную фазу, в артериальную фазу 12-190 ед.Н, в венозную фазу 12-185 ед.Н, в отсроченную фазу 12-81 ед.Н. По данным анализа фракционированных метанефринов (МН) суточной мочи: норметанефрин (НMH) 1394 мкг/сут (<390), MH 42,5 мкг/сут (<320). Проведена адреналэктомия с опухолью. При гистологическом исследовании верифицирована ФХЦ.

РЕЗУЛЬТАТЫ: для уточнения гистогенеза и клинического прогноза выполнено иммуногистохимическое исследование. Выявлено что клетки опухоли диффузно экспрессируют синаптофизин и очагово. хромогранин А. Подтверждена инвазия 1 сосуда капсулы, индекс пролиферации Кі-67 составил 20\%, также отмечалась диффузная цитоплазматическая мелкогранулированая экспрессия SDHB. Маркеры кортикальной дифференцировки (мелан А и ингибин альфа) в опухолевой ткани не экспрессировались при положительном внутреннем контроле. По шкале PASS 12 баллов (более 4 баллов — высокая вероятность рецидива), по шкале GAPP 8 баллов (низкодифференцированный тип). В послеоперационном периоде артериальное давление нормализовалось. Принимая во внимание результаты морфологического исследования, пациент находился под динамическим наблюдением с визитами к специалисту каждые 2 мес. Через 10 месяцев после хирургического лечения стал отмечать повышение АД мах до 180/110 мм рт.ст. При МСКТ брюшной полости в проекции удаленного правого надпочечника, парааортально и паракавально визуализированы мягкотканые новообразования неоднородной структуры размерами до 49х47 мм, с накоплением в артериальную фазу до 140 ед.Н. По данным анализа фракционированных МН суточной мочи: НМН 5535 мкг/сут (<390), МН 79,2 мкг/сут (<320). Результаты исследований свидетельствовали о прогрессировании опухоли.

ВЫВОдЫ: в представленном клиническом наблюдении применение шкал PASS и GAPP позволило определить высокий злокачественный потенциал ФХЦ, что потребовало активного наблюдения и позволило своевременно выявить рецидив заболевания и провести повторное потенциально радикальное хирургическое лечение.

КЛЮЧЕВЫЕ СЛОВА: феохромоцитома; артериальная гипертензия; адреналэктомия; иммуногистохимия. 